OPEN ACCESS

Edited by:

Qiwei Zhang,

Southern Medical University, China

Reviewed by:

Vitali Sintchenko,

University of Sydney, Australia Timothy James Wells,

The University of Queensland,

Australia

*Correspondence:

Yi-Wei Tang

tangy@mskcc.org

Specialty section:

This article was submitted to Infectious Diseases,

a section of the journal

Frontiers in Microbiology

Received: 18 July 2018 Accepted: 28 September 2018 Published: 18 October 2018

Citation:

Das S, Dunbar S and Tang Y-W (2018) Laboratory Diagnosis of Respiratory Tract Infections

in Children - the State of the Art.

Front. Microbiol. 9:2478.

doi: 10.3389/fmicb.2018.02478

\section{Laboratory Diagnosis of Respiratory Tract Infections in Children - the State of the Art}

\author{
Shubhagata Das ${ }^{1}$, Sherry Dunbar ${ }^{1}$ and Yi-Wei Tang ${ }^{2,3 *}$ \\ 1 Global Scientific Affairs, Luminex Corporation, Austin, TX, United States, ${ }^{2}$ Department of Laboratory Medicine, Memorial \\ Sloan Kettering Cancer Center, Weill Medical College of Cornell University, New York, NY, United States, ${ }^{3}$ Department \\ of Pathology and Laboratory Medicine, Memorial Sloan Kettering Cancer Center, Weill Medical College of Cornell University, \\ New York, NY, United States
}

In the pediatric population, respiratory infections are the most common cause of physician visits. Although many respiratory illnesses are self-limiting viral infections that resolve with time and supportive care, it can be critical to identify the causative pathogen at an early stage of the disease in order to implement effective antimicrobial therapy and infection control. Over the last few years, diagnostics for respiratory infections have evolved substantially, with the development of novel assays and the availability of updated tests for newer strains of pathogens. Newer laboratory methods are rapid, highly sensitive and specific, and are gradually replacing the conventional gold standards, although the clinical utility of these assays is still under evaluation. This article reviews the current laboratory methods available for testing for respiratory pathogens and discusses the advantages and disadvantages of each approach.

Keywords: respiratory tract infection, respiratory virus, pediatric population, respiratory infection diagnosis, molecular diagnostics

\section{INTRODUCTION}

Acute respiratory tract infections are one of the leading causes of childhood morbidity and mortality worldwide, and it has been estimated that globally, respiratory infections are responsible for about 2 million deaths in children between 0 and 5 years of age (Bryce et al., 2005; Kallander et al., 2008). Approximately $80 \%$ of these respiratory infection cases are caused by viral pathogens such as influenza A and B, respiratory syncytial virus (RSV) A and B, parainfluenza virus types $1-3$, adenovirus, rhinovirus, human metapneumovirus (hMPV), and others (Mahony, 2008). The non-specific clinical presentation of respiratory infections poses a considerable challenge to the differential diagnosis of these pathogens. Early and accurate diagnosis of the causative pathogens in respiratory infections is essential to administer appropriate antiviral or antibacterial therapy, initiate effective infection control measures, and reduce the length of hospital stay (Barenfanger et al., 2000; Byington et al., 2002; Akers et al., 2017). In the last decade, there has been a remarkable improvement in the diagnosis of respiratory pathogens with the availability of molecular and pointof-care (POC) testing. Although an increasing number of laboratories are adopting rapid molecular assays, conventional testing methods, such as culture and immunodiagnostics are still used. This review focuses on current laboratory methods for testing for respiratory pathogens, and discusses the advantages and disadvantages of each approach. 


\section{NON-MOLECULAR METHODS FOR DETECTION AND IDENTIFICATION OF RESPIRATORY PATHOGENS}

\section{Electron Microscopy}

Electron microscopy (EM) is one of the oldest direct examination methods that has been implemented for both clinical viral diagnosis and study of viral ultrastructure and pathogenesis in developed countries (Roingeard, 2008). Historically, EM has played an instrumental role in identifying novel viral strains in several outbreak situations, such as the coronavirus associated with the severe acute respiratory syndrome (SARS) outbreak (Falsey and Walsh, 2003; Ksiazek et al., 2003). However, despite several advantages, the use of EM has been limited in respiratory viral diagnosis as it is expensive, laborious, time-consuming, and has a greater turnaround time (approximately 3-16 h including specimen preparation), and is often insensitive when compared to other diagnostic methods (Goldsmith and Miller, 2009; Zhang et al., 2013). Additionally, EM requires strict control of experimental conditions, a high concentration of viral particles $\left(>10^{5} \mathrm{~L}^{-1}\right.$ ), and considerable technical skill and expertise for accurate analysis.

\section{Culture}

Detection of viruses by observing the cytopathic effect and hemadsorption in cell culture has been considered the "gold standard" for diagnosis of respiratory viral pathogens for decades. Viruses such as adenovirus, influenza A/B, RSV, and human parainfluenza viruses are the most common respiratory viruses that are isolated and detected by cell culture (Olsen et al., 1993; Winn and Koneman, 2006). The traditional tube culture method is advantageous for growing a wide variety of viruses, including novel or unknown viruses, but takes days and often weeks to provide results. Over the years, modified cell culture methods such as the centrifugation enhanced shell-vial method has reduced the turnaround time from 5 to 10 days to $24 \mathrm{~h}$ (Dilnessa and Zeleke, 2017). Shell-vial culture using combination cell lines allows simultaneous detection of multiple respiratory viruses and, as compared to conventional culture, has similar sensitivity for parainfluenza $1-3(87 \%$ vs. $83 \%)$ and influenza A/B (78\% vs. $75 \%$ ), and significantly higher sensitivity for RSV (73\% vs. $42 \%$ ) (LaSala et al., 2007). Despite these advantages, many clinically relevant viruses are difficult to grow in culture (such as rhinovirus and coronavirus) and may produce variable results (Ieven and Goossens, 1997; Hodinka, 2013). Additionally, multiple freezing and thawing of the samples prior to testing can reduce the viral titer, thus effecting the growth in culture. Therefore, as compared to molecular tests, both traditional tube and shell-vial culture methods are laborious, exhibit higher false negative rates, and have longer turnaround times, making viral culture less clinically relevant (Leland and Ginocchio, 2007; Ginocchio, 2007; Hematian et al., 2016).

Culture is also the gold standard for detection of atypical (bacterial) respiratory pathogens, which is followed by identification and antimicrobial susceptibility testing by various manual or automated methods (Tenover, 2011). Bacterial culture can also often be insensitive, especially when specimen adequacy is not determined by Gram stain or if specimens were collected after antibiotic exposure (ATS and IDSA, 2005; Woodhead et al., 2005; Harris et al., 2017). Bacterial culture is also labor intensive, requires substantial technical expertise, and has a typical turnaround time of $48-96 \mathrm{~h}$ if antibiotic susceptibility testing is performed, and can therefore be considered inadequate for optimal patient care and guiding effective antimicrobial therapy (Tenover, 2011).

\section{Antigen Detection Assays Rapid Immunoassays}

Rapid immunoassays (RIAs) can deliver test results in less than $30 \mathrm{~min}$ and are usually performed in the POC setting, thus allowing the test results to be incorporated into the clinical decision-making algorithm (Weinberg and Walker, 2005). Among the four primary RIA formats (latex agglutination, horizontal flow devices, lateral flow devices, and optical immunoassays), the lateral-flow immunoassay (LFIA) is the most versatile and popular immunochromatographic method. RIAs are relatively inexpensive, easy to perform, and most of them have waived status in the United States according to the Clinical Laboratory Improvement Act (CLIA) guidelines, thereby making them invaluable in outpatient clinics, primary care, emergency, and low resource settings (Ginocchio, 2007). Currently, commercially available RIAs are mostly limited to the detection of influenza A virus, influenza B virus, and RSV. Numerous studies have revealed that RIAs demonstrate overall poor sensitivity for influenza and RSV (44-95\%); however, they have a higher median specificity (90 to $95 \%$ ) as compared to cell culture (WHO, 2005; Leland and Ginocchio, 2007; Ginocchio, 2007).

In the pediatric population, commercially available immunoassays have demonstrated high sensitivity (93\%) for detection of RSV, and a systematic review of published studies has further revealed that the sensitivity of RSV RIAs is relatively higher for children (81\%) than adults (29\%) (Slinger et al., 2004; Chartrand et al., 2015). The higher sensitivity can be attributed to the fact that pediatric patients often shed higher titers of respiratory viruses and for a longer time as compared to adults (Englund et al., 1996; Kawai et al., 2007). Despite the overall lower sensitivity, RIAs have been deemed a valuable diagnostic tool in the emergency department as they can significantly decrease the length of stay, additional ancillary testing, and antibiotic prescriptions for those children who do test positive for influenza (Abanses et al., 2006; Blaschke et al., 2014).

\section{Direct Fluorescent Antibody Tests}

Direct fluorescent antibody (DFA) testing of nasopharyngeal wash specimens is considered a rapid and reliable method for detecting respiratory viral infections. Commercial DFA kits have demonstrated high sensitivity and specificity for multiple respiratory viruses such as hMPV (95 and 100\%), adenovirus (62 and 100\%), RSV (94 and 96\%), and parainfluenza viruses (88 and 99.7\%), although the results can be subjective and require technical expertise for accurate interpretation (Landry and Ferguson, 2000; Ohm-Smith et al., 2004; Rocholl et al., 2004; 
Aslanzadeh et al., 2008; Vinh et al., 2008). The high specificity (99-100\%) of DFA indicates that the test can be used as a reliable detection method, especially during the initial days of the illness, as was shown by Shafik et al. (2011) for RSV in children.

\section{Serological Tests}

Pathogen-specific antibodies typically appear about 2 weeks after the initial infection and can be detected by serological tests. Serological tests can successfully identify antibodies to most respiratory pathogens such as RSV, adenovirus, influenza $A$ and $B$, parainfluenza 1-3 virus, etc., and can detect mixed infections from hospitalized children suffering from acute respiratory infections, with the exception of infants for whom an antibody response is usually undetected (Hall et al., 1991; Chkhaidze et al., 2006). However, it has been reported that serological assays are significantly less sensitive for the detection of parainfluenza virus and adenovirus when compared to molecular methods, such as RT-PCR (Kuypers et al., 2006). Kuypers et al. (2006) found that RT-PCR detected 40\% more specimens from pediatric patients that were positive for at least one respiratory virus than were detected by fluorescent antibody assay (FA). FA testing, in addition to RT-PCR, is useful for epidemiological studies as it increases the probability of identifying acute viral infections and has been used for accurate assessment of respiratory viruses other than influenza in children (Sawatwong et al., 2012; Feikin et al., 2013; Zhang et al., 2017).

For bacteria, serological testing is particularly challenging, especially for identifying atypical bacterial agents such as Mycoplasma pneumoniae. With varied sensitivity (14\% $77 \%)$ and specificity $(49 \%$ - 97\%) as compared to PCR, serological testing has limited usefulness clinically (Beersma et al., 2005; Wellinghausen et al., 2006). The clinical utility of serologic tests is further limited because they require both acute and convalescent sera to monitor seroconversion or to identify a four-fold increase in antibody titer (Loeffelholz and Chonmaitree, 2010). Additionally, serological tests are not relevant for identifying frequently recurring viral infections as the serum IgM levels are lower due to repeated exposure to vaccines or circulating viruses. For optimal virus specific IgM testing, an acute-phase serum specimen should be obtained early in the course of illness (Dunn, 2015).

\section{NUCLEIC ACID AMPLIFICATION TESTS FOR DETECTION AND IDENTIFICATION OF RESPIRATORY PATHOGENS}

A wide variety of newer, nucleic acid amplification tests (NAATs) for the detection of respiratory pathogens are commercially available. These tests are listed in Table $\mathbf{1}$ and described below according to complexity and pathogen coverage. The accuracy of respiratory virus detection by molecular tests is not only dependent on their specific assay chemistry, but is also critically affected by the type, quantity, and quality of specimens collected (Dunn, 2015).
Several types of specimens can be used for detection of respiratory viruses, including: bronchoalveolar lavage (BAL), throat swab, nasopharyngeal (NP) washes, NP aspirates, lung aspirates, and NP swabs, although the appropriate specimen type depends on the specific patient population. For example, in pediatric patients, obtaining nasal washes and nasal aspirates is more technically challenging when compared to NP swabs, especially in severely ill children and in poor resource settings (Hammitt et al., 2012). It has been observed that for optimal test results, specimens should be collected within 3-5 days after onset of symptoms to ensure that the sample has a high concentration of virus particles, viral antigen or viral nucleic acids, should be transported to the testing laboratory in refrigerated condition $\left(2-8^{\circ} \mathrm{C}\right)$ in appropriate transport media, and testing should be performed within $48 \mathrm{~h}$ (Grys and Smith, 2009).

\section{High Complexity Multiplex Panel Assays}

Detection of respiratory pathogens by NAATs such as PCR, nucleic acid sequence-based amplification (NASBA), transcription mediated amplification (TMA), strand displacement amplification (SDA), loop-mediated isothermal amplification (LAMP), rolling circle amplification (RCA), etc., have gained immense popularity over the past decade. Large syndromic panels that can detect multiple pathogens simultaneously are beneficial for infection control, timely treatment decisions, and are substantially less expensive than detecting individual pathogens by monoplex real-time RT-PCR (Reijans et al., 2008; Schreckenberger and McAdam, 2015). These multiplex respiratory panels vary in terms of their complexity (high, moderate, or waived), throughput, pathogens detected, ability to subtype, instrumentation (batched or random access), workflow (stepwise or integrated sample-to-answer), ease of use, and time to result (several hours to minutes) (Caliendo, 2011; Krunic et al., 2011; Mahony et al., 2011). Studies have reported increased diagnostic yield ( $60 \%$ vs. $35 \%$ ) and considerably higher sensitivity $(80-100 \%)$ and specificity $(82-100 \%)$ for these assays when compared to conventional diagnostic methods such as DFA, viral isolation, and immunoassays (Reijans et al., 2008; Gharabaghi et al., 2011).

In comparison to other NAAT-based molecular methods, high-plex syndromic panels demonstrate comparable performance, although sensitivity and specificity might vary for individual targets (Sails et al., 2017). For example, greater than $99 \%$ specificity was observed for all targets except enterovirus/rhinovirus (94\%), and the overall sensitivity ranged between (83-100\%) when the NxTAG RPP and the Anyplex II RV16 assays were evaluated for detection of respiratory pathogens in hospitalized children (Brotons et al., 2016). Syndromic panels can also detect bacterial infections and viral-bacterial coinfections, which is vital for developing effective treatment plans and prevention strategies (Brealey et al., 2015; Brotons et al., 2016). However, for novel pathogen strains, such as Flu $\mathrm{A}(\mathrm{H} 1 \mathrm{~N} 1 \mathrm{pdm} 2009)$, panel tests often fail to identify the subtype and require confirmation by other tests (Bryce et al., 2012). 
TABLE 1 | Commercially available nucleic acid amplification assays for respiratory pathogens ${ }^{2}$.

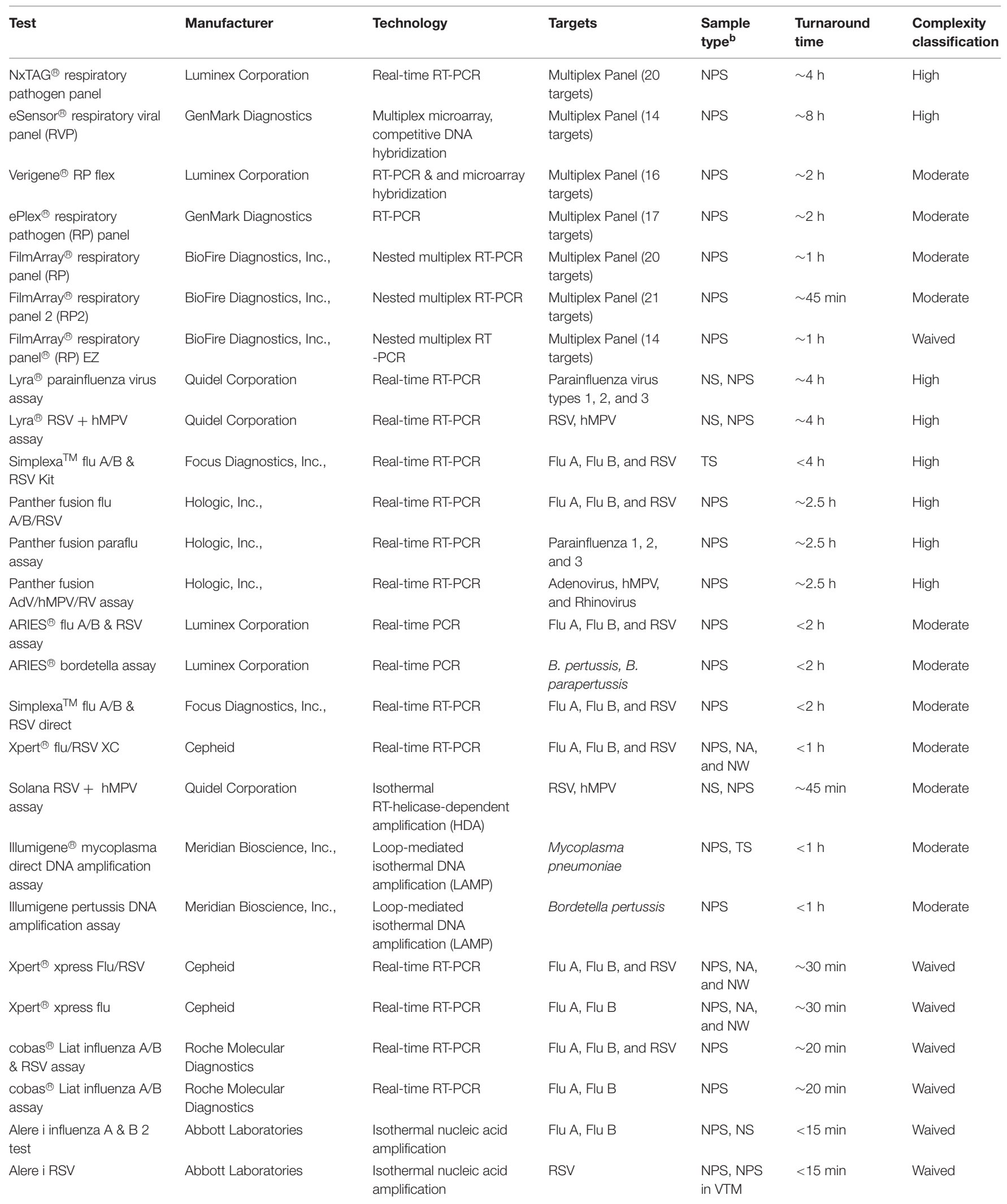

aFDA 510(k) Premarket Notification (FDA, 2018a). Retrieved from https://www.accessdata.fda.gov/scripts/cdrh/cfdocs/cfpmn/pmn.cfm; bNPS, nasopharyngeal swab; NS, nasal swab; NA, nasal aspirate; NW, nasal wash; TS, throat swab. ' Waived for direct NS, NPS, and NA/NW specimens; Moderate for NS and NA/NW specimens eluted in transport media. 
Syndromic respiratory panels with high sample throughput can usually run 1-96 specimens and are mostly classified as high complexity assays according to CLIA guidelines, which indicates that running these panel requires considerable knowledge, training, and experience (FDA, 2018b). It has been noted that these high throughput panels are ideal for batch testing, especially during outbreak situations and respiratory viral seasons when sample volumes are unusually high (Ginocchio et al., 2009; Crawford et al., 2010; Tang et al., 2016). Despite the advantages, implementation of high throughput panels can be challenging because of demanding sample preparation, processing, and result interpretation procedures, and the turnaround time varies from approximately 5-16 h (Beckmann and Hirsch, 2016).

\section{Moderate Complexity Multiplex Integrated Systems}

Moderate complexity sample-to-answer molecular test systems have gained immense popularity over the last few years because of their ease of use, faster turnaround time, and efficient workflow. Currently, the United States. FDA-cleared sampleto-answer test systems include both low-plex, targeted assays and high-plex panels that can detect multiple pathogens and pathogen classes. These tests usually have low to moderate sample throughput (e.g., 1-12 samples/run), but have faster turnaround times when compared to batched, high throughput, multiplex panels (Popowitch et al., 2013). The high-plex, sampleto-answer panels can detect up to 12-20 pathogens based on the panel size. Most of these qualitative assays allow random access that ensures that tests can be performed on demand, and the reactions occur in closed cassettes or cartridges, thereby minimizing the risk of contamination (Poritz et al., 2011; Popowitch and Miller, 2015).

Comparative studies evaluating the performance of these assays has reported more than 95\% agreement between different commercial platforms, and greater than $85 \%$ sensitivity and 99\% specificity in most cases (Babady et al., 2018). However, some high-plex sample-to-answer tests have demonstrated lower sensitivity for certain pathogens such as adenovirus (57\%), influenza A(H1N1pdm09) (73\%), and influenza B virus (77\%) (Popowitch et al., 2013). Cross-reactivity between adenovirus subtypes has also been reported for some assays, but the differentiation of these groups is not deemed clinically important for routine diagnosis (Lin et al., 2004; Gray et al., 2007).

Despite a short turnaround time of 1-2 $\mathrm{h}$, the clinical utility of these assays has been questioned since no statistically significant reduction in antibiotic prescription rates or mortality has been observed between patients who tested positive for a non-influenza virus and those who tested negative (48\% vs. $49 \%$ respectively) (Green et al., 2016). In some cases, a reduction in length of hospital stay (7 days vs. 9 days) and duration of isolation ( $75 \mathrm{~h}$ vs. $82 \mathrm{~h}$ ) was observed (Rogers et al., 2015; Akers et al., 2017). The primary concerns for the highplex sample-to-answer assays are high cost per test, and the inability to order customized, targeted panels (Ramanan et al., 2018). Multiplex assays such as the Verigene RP Flex panel
(Luminex) offer flexible testing and payment options by allowing users to order any combination of targets for an individual sample.

\section{Low-Plex Integrated Test Systems}

Low-plex integrated respiratory test systems typically target 1-4 pathogens per assay. Comparative studies evaluating the performance of these assays has reported more than 90\% concordance between different commercial platforms, and greater than 95\% sensitivity and specificity in most cases (Dugas et al., 2014; Selvaraju et al., 2014; McMullen et al., 2017). The total turnaround time per sample for these assays varies between $\sim 1-2 \mathrm{~h}$, and the number of samples that can be tested in a standard 8-h work shift depends on the number of instruments available in the testing laboratory (Ling et al., 2018). Smaller panels targeting a few pathogens that can be run independently (random access) or simultaneously (random batch) could be a viable option for controlling laboratory costs. Therefore, when considering among various testing options, it is recommended that in addition to clinical performance, other assay characteristics such as ease of use, panel composition, total turnaround time, hands-on time, and cost be considered before implementing an assay in various clinical settings (Juretschko et al., 2017).

\section{Waived Molecular Point-of-Care Tests}

Nucleic acid amplification based POC testing is relatively new in the realm of respiratory viral diagnosis and has generated contradicting opinions regarding their implementation and clinical utility. Molecular POC assays have extremely short turnaround times (<30 min), minimal hands-on time (1-2 min), and can be easily operated by non-laboratory staff members, thereby making them suitable for near patient implementation and testing (Brendish et al., 2015; Peters et al., 2017; Ling et al., 2018). Currently most of the United States. FDAcleared commercial POC assays are limited to the detection of influenza A/B and RSV viruses, with the exception of the FilmArray $^{\circledR}$ RP EZ (BioFire) which detects 14 targets (FDA, 2018c). Most of the studies evaluating the clinical performance of these assays have reported high sensitivity (87-100\%) and specificity $(>98 \%)$ for detecting influenza A/B and RSV in pediatric and adult patients (Bell et al., 2014; Nie et al., 2014; Popowitch and Miller, 2015; Gibson et al., 2017; Ling et al., 2018). However, these studies have also shown that the clinical performance varies and sensitivity is significantly low for influenza B (45.2-54.5\%); therefore, the results should be interpreted with caution and additional confirmatory testing should be considered, depending on the clinical circumstances (Jokela et al., 2015; Busson et al., 2017; Davis et al., 2017). Molecular POC tests can be costly and more prone to incorrect results and contamination, as the assays are usually performed by non-laboratory personnel and poor training, failure to follow manufacturer's instructions, and failure to perform adequate quality control might affect the accuracy of results (Azar and Landry, 2018). Currently, more evidence-based studies are required to understand the overall clinical utility of molecular POC testing, since no correlation has been observed 
between implementation of these tests and reduction of antibiotic usage or hospital length of stay (Andrews et al., 2017; Brendish et al., 2017).

\section{CONCLUDING REMARKS}

Molecular testing has considerably improved the diagnosis of respiratory pathogens and is being considered as the new "gold standard". Although these tests have gained immense popularity, factors such as the patient population (adult, pediatric, and immunocompromised), the size of the laboratory, the purpose of testing (routine or urgent care), and cost/benefit ratio should be considered before implementing a particular assay. For example, during the 2012-2013 influenza outbreak, two nosocomial cases were identified at Memorial Sloan Kettering Cancer Center on January 1, 2013. Despite implementing appropriate infection control measures, five more cases were identified few days later on January 7, 2013. A delay in reporting the positive influenza result was observed as the laboratory was following the routine diagnostic algorithm, and this might have resulted in the subsequent cases of nosocomial infection. Following the 2012-2013 outbreak, the laboratory implemented the FilmArray ${ }^{\circledR} \mathrm{RP}$ for routine diagnosis and the Xpert $^{\circledR}$ Xpress Flu/RSV, a rapid molecular POC system, for seasonal use during epidemics and pandemics because the needs for routine testing and urgent care are completely different in these situations. Molecular tests are highly sensitive and specific and result in higher pathogen detection, and therefore physicians and attending clinicians should evaluate test results before initiating a particular course of treatment. Rapid molecular tests are best suited for routine diagnosis and outbreak situations; whereas, conventional methods, such as cell culture or EM could be considered for identifying novel strains of the pathogens.

\section{REFERENCES}

Abanses, J. C., Dowd, M. D., Simon, S. D., and Sharma, V. (2006). Impact of rapid influenza testing at triage on management of febrile infants and young children. Pediatr. Emerg. Care 22, 145-149. doi: 10.1097/01.pec. 0000202454.19237.b0

Akers, I. E., Weber, R., Sax, H., Boni, J., Trkola, A., and Kuster, S. P. (2017). Influence of time to diagnosis of severe influenza on antibiotic use, length of stay, isolation precautions, and mortality: a retrospective study. Influenza Other Respir. Viruses 11, 337-344. doi: 10.1111/irv.12454

Andrews, D., Chetty, Y., Cooper, B. S., Virk, M., Glass, S. K., Letters, A., et al. (2017). Multiplex PCR point of care testing versus routine, laboratory-based testing in the treatment of adults with respiratory tract infections: a quasi-randomised study assessing impact on length of stay and antimicrobial use. BMC Infect. Dis. 17:671. doi: 10.1186/s12879-017-2784-Z

Aslanzadeh, J., Zheng, X., Li, H., Tetreault, J., Ratkiewicz, I., Meng, S., et al. (2008). Prospective evaluation of rapid antigen tests for diagnosis of respiratory syncytial virus and human metapneumovirus infections. J. Clin. Microbiol. 46, 1682-1685. doi: $10.1128 / \mathrm{jcm} .00008-08$

ATS and IDSA (2005). Guidelines for the management of adults with hospitalacquired, ventilator-associated, and healthcare-associated pneumonia. Am. J. Respir. Crit. Care Med. 171, 388-416. doi: 10.1164/rccm.200405-644ST

Azar, M. M., and Landry, M. L. (2018). Detection of influenza A and B viruses and respiratory syncytial virus using CLIA-waived point-of-care assays: a paradigm
With the introduction of new technologies, newer assays will be developed for respiratory and other pathogens. For example, using NP swab specimens from children, Graf et al. (2016) demonstrated that untargeted next-generation sequencing-based metagenomics (mNGS) testing had excellent agreement (93\%) with multiplex molecular panels and detected more viruses that were either not targeted by the panel or missed due to highly divergent genome sequences. They concluded that mNGS can be used for accurate and unbiased detection of expected or unexpected pathogens. Implementing mNGS downstream of rapid molecular panel testing has been proven useful for characterizing the nature of hospital-associated transmission events and directing the control strategies in pediatric populations during outbreak situations (Greninger et al., 2017). However, diagnostic implementation of NGS is currently limited by incomplete understanding of analytical performance, high cost of the system and complexity of sequence data analysis. Future research will be necessary to focus on demonstrating the clinical utility of these new and upcoming assays in various patient populations and in resource limited settings.

\section{AUTHOR CONTRIBUTIONS}

All authors listed have made a substantial, direct and intellectual contribution to the work, and approved it for publication.

\section{FUNDING}

The study was supported in part by a research agreement between the Memorial Sloan Kettering Cancer Center and the Luminex Corporation (SK2013-0929) and by an NIH/NCI Cancer Center Support Grant P30 (CA008748).

shift to molecular tests. J. Clin. Microbiol. 56:e0367-18. doi: $10.1128 / \mathrm{jcm}$. 00367-18

Babady, N. E., England, M. R., Jurcic Smith, K. L., He, T., Wijetunge, D. S., Tang, Y. W., et al. (2018). Multicenter evaluation of the ePlex respiratory pathogen panel for the detection of viral and bacterial respiratory tract pathogens in nasopharyngeal swabs. J. Clin. Microbiol. 56, e01658-17. doi: 10.1128/jcm. 01658-17

Barenfanger, J., Drake, C., Leon, N., Mueller, T., and Troutt, T. (2000). Clinical and financial benefits of rapid detection of respiratory viruses: an outcomes study. J. Clin. Microbiol. 38, 2824-2828.

Beckmann, C., and Hirsch, H. H. (2016). Comparing luminex NxTAGrespiratory pathogen panel and RespiFinder-22 for multiplex detection of respiratory pathogens. J. Med. Virol. 88, 1319-1324. doi: 10.1002/ jmv.24492

Beersma, M. F., Dirven, K., van Dam, A. P., Templeton, K. E., Claas, E. C., and Goossens, H. (2005). Evaluation of 12 commercial tests and the complement fixation test for Mycoplasma pneumoniae-specific immunoglobulin G (IgG) and IgM antibodies, with PCR used as the “gold standard”. J. Clin. Microbiol. 43, 2277-2285. doi: 10.1128/jcm.43.5. 2277-2285.2005

Bell, J., Bonner, A., Cohen, D. M., Birkhahn, R., Yogev, R., Triner, W., et al. (2014). Multicenter clinical evaluation of the novel Alere ${ }^{\text {TM }}$ I Influenza A\&B isothermal nucleic acid amplification test. J. Clin. Virol. 61, 81-86. doi: 10.1016/j.jcv.2014. 06.001 
Blaschke, A. J., Shapiro, D. J., Pavia, A. T., Byington, C. L., Ampofo, K., Stockmann, C., et al. (2014). A national study of the impact of rapid influenza testing on clinical care in the emergency department. J. Pediatr. Infect Dis. Soc. 3, 112-118. doi: 10.1093/jpids/pit071

Brealey, J. C., Sly, P. D., Young, P. R., and Chappell, K. J. (2015). Viral bacterial coinfection of the respiratory tract during early childhood. FEMS Microbiol. Lett. 362:fnv062. doi: 10.1093/femsle/fnv062

Brendish, N. J., Malachira, A. K., Armstrong, L., Houghton, R., Aitken, S., Nyimbili, E., et al. (2017). Routine molecular point-of-care testing for respiratory viruses in adults presenting to hospital with acute respiratory illness (ResPOC): a pragmatic, open-label, randomised controlled trial. Lancet Respir. Med. 5, 401-411. doi: 10.1016/S2213-2600 (17)30120-0

Brendish, N. J., Schiff, H. F., and Clark, T. W. (2015). Point-of-care testing for respiratory viruses in adults: the current landscape and future potential. J. Infect. 71, 501-510. doi: 10.1016/j.jinf.2015.07.008

Brotons, P., Henares, D., Latorre, I., Cepillo, A., Launes, C., and MunozAlmagro, C. (2016). Comparison of NxTAG respiratory pathogen panel and anyplex II RV16 tests for multiplex detection of respiratory pathogens in hospitalized children. J. Clin. Microbiol. 54, 2900-2904. doi: 10.1128/jcm. 01243-16

Bryce, J., Boschi-Pinto, C., Shibuya, K., and Black, R. E. (2005). WHO estimates of the causes of death in children. Lancet 365, 1147-1152. doi: 10.1016/s01406736(05)71877-8

Bryce, L., Koenig, M., and Jerke, K. H. (2012). A large-scale study of respiratory virus infection over 2 years using the Luminex xTAGRVP assay. Mil. Med. 177, 1533-1538. doi: 10.7205/ MILMED-D-12-00277

Busson, L., Mahadeb, B., De Foor, M., Vandenberg, O., and Hallin, M. (2017). Contribution of a rapid influenza diagnostic test to manage hospitalized patients with suspected influenza. Diagn. Microbiol. Infect. Dis. 87, 238-242. doi: 10.1016/j.diagmicrobio.2016.11.015

Byington, C. L., Castillo, H., Gerber, K., Daly, J. A., Brimley, L. A., Adams, S., et al. (2002). The effect of rapid respiratory viral diagnostic testing on antibiotic use in a children's hospital. Arch. Pediatr. Adolesc. Med. 156, 1230-1234. doi: 10.1001/archpedi.156.12.1230

Caliendo, A. M. (2011). Multiplex PCR and emerging technologies for the detection of respiratory pathogens. Clin. Infect. Dis. 52(Suppl. 4), S326-S330. doi: $10.1093 / \mathrm{cid} / \mathrm{cir} 047$

Chartrand, C., Tremblay, N., Renaud, C., and Papenburg, J. (2015). Diagnostic accuracy of rapid antigen detection tests for respiratory syncytial virus infection: systematic review and meta-analysis. J. Clin. Microbiol. 53, 3738-3749. doi: $10.1128 / \mathrm{jcm} .01816-15$

Chkhaidze, I., Manjavidze, N., and Nemsadze, K. (2006). Serodiagnosis of acute respiratory infections in children in Georgia. Indian J. Pediatr. 73, 569-572. doi: 10.1007/BF02759919

Crawford, J. M., Stallone, R., Zhang, F., Gerolimatos, M., Korologos, D. D., Sweetapple, C., et al. (2010). Laboratory surge response to pandemic (H1N1) 2009 outbreak, New York City metropolitan area, USA. Emerg. Infect. Dis. 16, 8-13. doi: 10.3201/eid1601.091167

Davis, S., Allen, A. J., O'Leary, R., Power, M., Price, D. A., Simpson, A. J., et al. (2017). Diagnostic accuracy and cost analysis of the Alere ${ }^{\mathrm{TM}}$ I Influenza $\mathrm{A} \& \mathrm{~B}$ near-patient test using throat swabs. J. Hosp. Infect. 97, 301-309. doi: $10.1016 /$ j.jhin.2017.05.017

Dilnessa, T., and Zeleke, H. (2017). Cell culture, cytopathic effect and immunofluorescence diagnosis of viral infection. J. Microbiol. Mod. Tech. 2, $102-110$.

Dugas, A. F., Valsamakis, A., Gaydos, C. A., Forman, M., Hardick, J., Kidambi, P., et al. (2014). Evaluation of the Xpert Flu rapid PCR assay in highrisk emergency department patients. J. Clin. Microbiol. 52, 4353-4355. doi: $10.1128 / \mathrm{jcm} .02343-14$

Dunn, J. J. (2015). “Specimen collection, transport, and processing: virology," in Manual of Clinical Microbiology, 11th Edn, eds J. H. Jorgensen, M. A. Pfaller, K. C. Carroll, G. Funke, S. S. Richter, M. L. Landry, et al. (Washington, DC: American Society of Microbiology), 1405-1421.

Englund, J. A., Piedra, P. A., Jewell, A., Patel, K., Baxter, B. B., and Whimbey, E. (1996). Rapid diagnosis of respiratory syncytial virus infections in immunocompromised adults. J. Clin. Microbiol. 34, 1649-1653.
Falsey, A. R., and Walsh, E. E. (2003). Novel coronavirus and severe acute respiratory syndrome. Lancet 361, 1312-1313. doi: 10.1016/s0140-6736(03) 13084-X

FDA (2018a). 510(k) Premarket Notification [Online]. Available at: https: //www.accessdata.fda.gov/scripts/cdrh/cfdocs/cfpmn/pmn.cfm [accessed June $24,2018]$.

FDA (2018b). CLIA Categorizations [Online]. Available at: https://www.fda.gov/ MedicalDevices/DeviceRegulationandGuidance/IVDRegulatoryAssistance/ ucm393229.htm [accessed June 24, 2018].

FDA (2018c). Nucleic Acid Based Tests [Online]. Available at: https://www.fda. gov/MedicalDevices/ProductsandMedicalProcedures/InVitroDiagnostics/ ucm330711.htm [accessed June 24, 2018].

Feikin, D. R., Njenga, M. K., Bigogo, G., Aura, B., Gikunju, S., Balish, A., et al. (2013). Additional diagnostic yield of adding serology to PCR in diagnosing viral acute respiratory infections in Kenyan patients 5 years of age and older. Clin. Vaccine Immunol. 20, 113-114. doi: 10.1128/cvi.00325-12

Gharabaghi, F., Hawan, A., Drews, S. J., and Richardson, S. E. (2011). Evaluation of multiple commercial molecular and conventional diagnostic assays for the detection of respiratory viruses in children. Clin. Microbiol. Infect. 17, 19001906. doi: 10.1111/j.1469-0691.2011.03529.x

Gibson, J., Schechter-Perkins, E. M., Mitchell, P., Mace, S., Tian, Y., Williams, K., et al. (2017). Multi-center evaluation of the cobas ${ }^{\circledR}$ Liat ${ }^{\circledR}$ Influenza A/B \& RSV assay for rapid point of care diagnosis. J. Clin. Virol. 95, 5-9. doi: 10.1016/j.jcv. 2017.08.004

Ginocchio, C. C. (2007). Detection of respiratory viruses using non-molecular based methods. J. Clin. Virol. 40(Suppl. 1), S11-S14. doi: 10.1016/s13866532(07)70004-5

Ginocchio, C. C., Zhang, F., Manji, R., Arora, S., Bornfreund, M., Falk, L., et al. (2009). Evaluation of multiple test methods for the detection of the novel 2009 influenza A (H1N1) during the New York City outbreak. J. Clin. Virol. 45, 191-195. doi: 10.1016/j.jcv.2009.06.005

Goldsmith, C. S., and Miller, S. E. (2009). Modern uses of electron microscopy for detection of viruses. Clin. Microbiol. Rev. 22, 552-563. doi: 10.1128/cmr.00027-09

Graf, E. H., Simmon, K. E., Tardif, K. D., Hymas, W., Flygare, S., Eilbeck, K., et al. (2016). Unbiased detection of respiratory viruses using RNA-seq-based metagenomics: a systematic comparison to a commercial PCR panel. J. Clin. Microbiol. 54, 1000-1007. doi: 10.1128/JCM.03060-15

Gray, G. C., McCarthy, T., Lebeck, M. G., Schnurr, D. P., Russell, K. L., Kajon, A. E., et al. (2007). Genotype prevalence and risk factors for severe clinical adenovirus infection, United States 2004-2006. Clin. Infect. Dis. 45, 1120-1131. doi: 10.1086/522188

Green, D. A., Hitoaliaj, L., Kotansky, B., Campbell, S. M., and Peaper, D. R. (2016). Clinical utility of on-demand multiplex respiratory pathogen testing among adult outpatients. J. Clin. Microbiol. 54, 2950-2955. doi: 10.1128/jcm. 01579-16

Greninger, A. L., Waghmare, A., Adler, A., Qin, X., Crowley, J. L., Englund, J. A., et al. (2017). Rule-out outbreak: 24-hour metagenomic next-generation sequencing for characterizing respiratory virus source for infection prevention. J. Pediatr. Infect. Dis. Soc. 6, 168-172. doi: 10.1093/jpids/pix019

Grys, T. E., and Smith, T. F. (2009). "Specimen requirements: selection, collection, transport, and processing," in Clinical Virology Manual, 4th Edn, eds M. J. Loeffelholz, R. L. Hodinka, S. A. Young, and B. A. Pinsky (Washington, DC: American Society of Microbiology), 18-35.

Hall, C. B., Walsh, E. E., Long, C. E., and Schnabel, K. C. (1991). Immunity to and frequency of reinfection with respiratory syncytial virus. J. Infect. Dis. 163, 693-698. doi: 10.1093/infdis/163.4.693

Hammitt, L. L., Murdoch, D. R., Scott, J. A. G., Driscoll, A., Karron, R. A., Levine, O. S., et al. (2012). Specimen collection for the diagnosis of pediatric pneumonia. Clin. Infect. Dis. 54(Suppl. 2), S132-S139. doi: 10.1093/cid/cir1068

Harris, A. M., Bramley, A. M., Jain, S., Arnold, S. R., Ampofo, K., Self, W. H., et al. (2017). Influence of antibiotics on the detection of bacteria by culturebased and culture-independent diagnostic tests in patients hospitalized with community-acquired pneumonia. Open Forum Infect. Dis. 4:ofx014. doi: 10. 1093/ofid/ofx014

Hematian, A., Sadeghifard, N., Mohebi, R., Taherikalani, M., Nasrolahi, A., Amraei, M., et al. (2016). Traditional and modern cell culture in virus diagnosis. Osong Public Health Res. Perspect. 7, 77-82. doi: 10.1016/j.phrp.2015.11.011 
Hodinka, R. L. (2013). Point: is the era of viral culture over in the clinical microbiology laboratory? J. Clin. Microbiol. 51, 2-4. doi: $10.1128 / \mathrm{jcm}$ .02593-12

Ieven, M., and Goossens, H. (1997). Relevance of nucleic acid amplification techniques for diagnosis of respiratory tract infections in the clinical laboratory. Clin. Microbiol. Rev. 10, 242-256.

Jokela, P., Vuorinen, T., Waris, M., and Manninen, R. (2015). Performance of the Alere I influenza $\mathrm{A} \& \mathrm{~B}$ assay and mariPOC test for the rapid detection of influenza A and B viruses. J. Clin. Virol. 70, 72-76. doi: 10.1016/j.jcv.2015. 07.294

Juretschko, S., Mahony, J., Buller, R. S., Manji, R., Dunbar, S., Walker, K., et al. (2017). Multicenter clinical evaluation of the luminex Aries Flu A/B \& RSV assay for pediatric and adult respiratory tract specimens. J. Clin. Microbiol. 55, 2431-2438. doi: $10.1128 / \mathrm{jcm} .00318-17$

Kallander, K., Hildenwall, H., Waiswa, P., Galiwango, E., Peterson, S., and Pariyo, G. (2008). Delayed care seeking for fatal pneumonia in children aged under five years in Uganda: a case-series study. Bull. World Health Organ. 86, 332-338. doi: 10.2471/BLT.07.049353

Kawai, N., Ikematsu, H., Iwaki, N., Kawashima, T., Maeda, T., Mitsuoka, S., et al. (2007). Longer virus shedding in influenza $B$ than in influenza $A$ among outpatients treated with oseltamivir. J. Infect. 55, 267-272. doi: 10.1016/j.jinf. 2007.05.176

Krunic, N., Merante, F., Yaghoubian, S., Himsworth, D., and Janeczko, R. (2011). Advances in the diagnosis of respiratory tract infections: role of the Luminex xTAG respiratory viral panel. Ann. N. Y. Acad. Sci. 1222, 6-13. doi: 10.1111/j. 1749-6632.2011.05964.x

Ksiazek, T. G., Erdman, D., Goldsmith, C. S., Zaki, S. R., Peret, T., Emery, S., et al. (2003). A novel coronavirus associated with severe acute respiratory syndrome. N. Engl. J. Med. 348, 1953-1966. doi: 10.1056/ NEJMoa030781

Kuypers, J., Wright, N., Ferrenberg, J., Huang, M. L., Cent, A., Corey, L., et al. (2006). Comparison of real-time PCR assays with fluorescent-antibody assays for diagnosis of respiratory virus infections in children. J. Clin. Microbiol. 44, 2382-2388. doi: 10.1128/jcm.00216-06

Landry, M. L., and Ferguson, D. (2000). SimulFluor respiratory screen for rapid detection of multiple respiratory viruses in clinical specimens by immunofluorescence staining. J. Clin. Microbiol. 38, 708-711.

LaSala, P. R., Bufton, K. K., Ismail, N., and Smith, M. B. (2007). Prospective comparison of $\mathrm{R}-\mathrm{mix}^{\mathrm{TM}}$ shell vial system with direct antigen tests and conventional cell culture for respiratory virus detection. J. Clin. Virol. 38, 210-216. doi: 10.1016/j.jcv.2006.12.015

Leland, D. S., and Ginocchio, C. C. (2007). Role of cell culture for virus detection in the age of technology. Clin. Microbiol. Rev. 20, 49-78. doi: $10.1128 / \mathrm{cmr} .00002-06$

Lin, B., Vora, G. J., Thach, D., Walter, E., Metzgar, D., Tibbetts, C., et al. (2004). Use of oligonucleotide microarrays for rapid detection and serotyping of acute respiratory disease-associated adenoviruses. J. Clin. Microbiol. 42, 3232-3239. doi: $10.1128 / \mathrm{jcm} \cdot 42.7 .3232-3239.2004$

Ling, L., Kaplan, S. E., Lopez, J. C., Stiles, J., Lu, X., and Tang, Y. W. (2018). Parallel validation of three molecular devices for simultaneous detection and identification of influenza A and B and respiratory syncytial viruses. J. Clin. Microbiol. 56:e01691-17. doi: 10.1128/jcm.01691-17

Loeffelholz, M., and Chonmaitree, T. (2010). Advances in diagnosis of respiratory virus infections. Int. J. Microbiol. 2010:126049. doi: 10.1155/ $2010 / 126049$

Mahony, J. B. (2008). Detection of respiratory viruses by molecular methods. Clin. Microbiol. Rev. 21, 716-747. doi: 10.1128/cmr.00037-07

Mahony, J. B., Petrich, A., and Smieja, M. (2011). Molecular diagnosis of respiratory virus infections. Crit. Rev. Clin. Lab. Sci. 48, 217-249. doi: 10.3109/ 10408363.2011.640976

McMullen, P., Boonlayangoor, S., Charnot-Katsikas, A., Beavis, K. G., and Tesic, V. (2017). The performance of Luminex ARIES ${ }^{\circledR}$ Flu A/B \& RSV and Cepheid Xpert $^{\circledR}$ Flu/RSV XC for the detection of influenza A, influenza B, and respiratory syncytial virus in prospective patient samples. J. Clin. Virol. 95, 84-85. doi: 10.1016/j.jcv.2017.08.018

Nie, S., Roth, R. B., Stiles, J., Mikhlina, A., Lu, X., Tang, Y. W., et al. (2014). Evaluation of Alere I influenza A\&B for rapid detection of influenza viruses A and B. J. Clin. Microbiol. 52, 3339-3344. doi: 10.1128/jcm.01132- 14
Ohm-Smith, M. J., Nassos, P. S., and Haller, B. L. (2004). Evaluation of the Binax NOW, BD Directigen, and BD Directigen EZ assays for detection of respiratory syncytial virus. J. Clin. Microbiol. 42, 2996-2999. doi: 10.1128/jcm.42.7.29962999.2004

Olsen, M. A., Shuck, K. M., Sambol, A. R., Flor, S. M., O’Brien, J., and Cabrera, B. J. (1993). Isolation of seven respiratory viruses in shell vials: a practical and highly sensitive method. J. Clin. Microbiol. 31, 422-425.

Peters, R. M., Schnee, S. V., Tabatabai, J., Schnitzler, P., and Pfeil, J. (2017). Evaluation of Alere I RSV for rapid detection of respiratory syncytial virus in children hospitalized with acute respiratory tract infection. J. Clin. Microbiol. 55, 1032-1036. doi: $10.1128 / \mathrm{jcm} .02433-16$

Popowitch, E. B., and Miller, M. B. (2015). Performance characteristics of Xpert Flu/RSV XC assay. J. Clin. Microbiol. 53, 2720-2721. doi: 10.1128/jcm.00972-15

Popowitch, E. B., O'Neill, S. S., and Miller, M. B. (2013). Comparison of the Biofire FilmArray RP, Genmark eSensor RVP, Luminex xTAG RVPv1, and Luminex xTAG RVP fast multiplex assays for detection of respiratory viruses. J. Clin. Microbiol. 51, 1528-1533. doi: 10.1128/jcm.03368-12

Poritz, M. A., Blaschke, A. J., Byington, C. L., Meyers, L., Nilsson, K., Jones, D. E., et al. (2011). FilmArray, an automated nested multiplex PCR system for multipathogen detection: development and application to respiratory tract infection. PLoS One 6:e26047. doi: 10.1371/journal.pone.0026047

Ramanan, P., Bryson, A. L., Binnicker, M. J., Pritt, B. S., and Patel, R. (2018). Syndromic panel-based testing in clinical microbiology. Clin. Microbiol. Rev. 31:e00024-17. doi: 10.1128/cmr.00024-17

Reijans, M., Dingemans, G., Klaassen, C. H., Meis, J. F., Keijdener, J., Mulders, B., et al. (2008). RespiFinder: a new multiparameter test to differentially identify fifteen respiratory viruses. J. Clin. Microbiol. 46, 1232-1240. doi: 10.1128/jcm. 02294-07

Rocholl, C., Gerber, K., Daly, J., Pavia, A. T., and Byington, C. L. (2004). Adenoviral infections in children: the impact of rapid diagnosis. Pediatrics 113(1 Pt 1), e51-e56.

Rogers, B. B., Shankar, P., Jerris, R. C., Kotzbauer, D., Anderson, E. J., Watson, J. R., et al. (2015). Impact of a rapid respiratory panel test on patient outcomes. Arch. Pathol. Lab. Med. 139, 636-641. doi: 10.5858/arpa.2014-0257-OA

Roingeard, P. (2008). Viral detection by electron microscopy: past, present and future. Biol. Cell 100, 491-501. doi: 10.1042/bc20070173

Sails, A. D., Eltringham, G., Valappil, M., Waugh, S., and Saunders, D. (2017). Comparison of the Luminex NxTAG respiratory pathogen panel and a multiplex in-house real-time PCR panel for the detection of respiratory viruses in symptomatic patients. J. Med. Microbiol. doi: 10.1099/jmm.0.000562 [Epub ahead of print].

Sawatwong, P., Chittaganpitch, M., Hall, H., Peruski, L. F., Xu, X., Baggett, H. C., et al. (2012). Serology as an adjunct to polymerase chain reaction assays for surveillance of acute respiratory virus infections. Clin. Infect. Dis. 54, 445-446. doi: $10.1093 / \mathrm{cid} / \mathrm{cir} 710$

Schreckenberger, P. C., and McAdam, A. J. (2015). Point-counterpoint: large multiplex PCR panels should be first-line tests for detection of respiratory and intestinal pathogens. J. Clin. Microbiol. 53, 3110-3115. doi: 10.1128/jcm.003 $82-15$

Selvaraju, S. B., Bambach, A. V., Leber, A. L., Patru, M.-M., Patel, A., and Menegus, M. A. (2014). Comparison of the Simplexa ${ }^{\mathrm{TM}}$ Flu A/B \& RSV kit (nucleic acid extraction-dependent assay) and the Prodesse ProFlu $+{ }^{\mathrm{TM}}$ assay for detecting influenza and respiratory syncytial viruses. Diagn. Microbiol. Infect. Dis. 80, 50-52. doi: 10.1016/j.diagmicrobio.2013.11.015

Shafik, C. F., Mohareb, E. W., and Youssef, F. G. (2011). Comparison of direct fluorescence assay and real-time rt-PCR as diagnostics for respiratory syncytial virus in young children. J. Trop. Med. 2011:781919. doi: 10.1155/ 2011/781919

Slinger, R., Milk, R., Gaboury, I., and Diaz-Mitoma, F. (2004). Evaluation of the QuickLab RSV test, a new rapid lateral-flow immunoassay for detection of respiratory syncytial virus antigen. J. Clin. Microbiol. 42, 3731-3733. doi: 10. $1128 / \mathrm{jcm} .42 .8 .3731-3733.2004$

Tang, Y. W., Gonsalves, S., Sun, J. Y., Stiles, J., Gilhuley, K. A., Mikhlina, A., et al. (2016). Clinical evaluation of the Luminex NxTAG respiratory pathogen panel. J. Clin. Microbiol. 54, 1912-1914. doi: 10.1128/jcm.00482-16

Tenover, F. C. (2011). Developing molecular amplification methods for rapid diagnosis of respiratory tract infections caused by bacterial pathogens. Clin. Infect. Dis. 52(Suppl. 4), S338-S345. doi: 10.1093/cid/cir049 
Vinh, D. C., Newby, D., Charest, H., and McDonald, J. (2008). Evaluation of a commercial direct fluorescent-antibody assay for human metapneumovirus in respiratory specimens. J. Clin. Microbiol. 46, 1840-1841. doi: 10.1128/jcm. 01554-07

Weinberg, A., and Walker, M. L. (2005). Evaluation of three immunoassay kits for rapid detection of influenza virus A and B. Clin. Diagn. Lab. Immunol. 12, 367-370. doi: 10.1128/cdli.12.3.367-370.2005

Wellinghausen, N., Straube, E., Freidank, H., von Baum, H., Marre, R., and Essig, A. (2006). Low prevalence of Chlamydia pneumoniae in adults with communityacquired pneumonia. Int. J. Med. Microbiol. 296, 485-491. doi: 10.1016/j.ijmm. 2006.05.003

WHO (2005). WHO Recommendations on the use of Rapid Testing for Influenza Diagnosis [Online]. Available at: http://www.who.int/influenza/ resources/documents/RapidTestInfluenza_WebVersion.pdf [accessed June 24, 2018].

Winn, W. C., and Koneman, E. W. (2006). Koneman's Color Atlas and Textbook of Diagnostic Microbiology. Philadelphia, PA: Lippincott Williams \& Wilkins.

Woodhead, M., Blasi, F., Ewig, S., Huchon, G., Ieven, M., Ortqvist, A., et al. (2005). Guidelines for the management of adult lower respiratory tract infections. Eur. Respir. J. 26, 1138-1180. doi: 10.1183/09031936. 05.00055705
Zhang, Y., Hung, T., Song, J., and He, J. (2013). Electron microscopy: essentials for viral structure, morphogenesis and rapid diagnosis. Sci. China Life Sci. 56, 421-430. doi: 10.1007/s11427-013-4476-2

Zhang, Y., Sakthivel, S. K., Bramley, A., Jain, S., Haynes, A., Chappell, J. D., et al. (2017). Serology enhances molecular diagnosis of respiratory virus infections other than influenza in children and adults hospitalized with community-acquired pneumonia. J. Clin. Microbiol. 55, 79-89. doi: $10.1128 / \mathrm{jcm} .01701-16$

Conflict of Interest Statement: SDa and SDu are employees of Luminex Corporation.

The remaining author declares that the research was conducted in the absence of any commercial or financial relationships that could be construed as a potential conflict of interest.

Copyright $\odot 2018$ Das, Dunbar and Tang. This is an open-access article distributed under the terms of the Creative Commons Attribution License (CC BY). The use, distribution or reproduction in other forums is permitted, provided the original author(s) and the copyright owner(s) are credited and that the original publication in this journal is cited, in accordance with accepted academic practice. No use, distribution or reproduction is permitted which does not comply with these terms. 